\title{
ESTIMATIVA DAS CONDIÇÕES DE CONFORTO TÉRMICO PARA AVICULTURA DE POSTURA USANDO A TEORIA DOS CONJUNTOS FUZZY
}

\author{
HENRIQUE L. DE OLIVEIRA ${ }^{1}$, MARIANGELA AMENDOLA², \\ IRENILZA DE A. NÄ̈̈̈s
}

RESUMO: Neste trabalho, foi utilizada uma ferramenta matemática promissora na análise de sistemas e/ou processos, particularmente na área de produção animal. Essa ferramenta é a desenvolvida segundo a abordagem da teoria dos Conjuntos Fuzzy e, neste caso específico, permitiu a análise da composição das variáveis climáticas independentes, como temperatura de bulbo seco e umidade relativa do ar, que influenciam na variável dependente denominada conforto térmico das aves. Foi realizada a construção de regras baseadas na intuição humana, segundo o conhecimento de especialistas da área, a partir do que é possível simular cenários distintos para o suporte à decisão de construção de galpões para abrigo a animais. Neste trabalho, foi estimado o conforto térmico para alojamento de aves poedeiras em produção. Os resultados foram analisados, usando-se o ambiente de computação científica MATLAB 6.5, o que pode ser realizado iterativamente a cada cenário gerado. Com base nos resultados obtidos, pode-se analisar as condições de conforto para distintas composições das variáveis de entrada.

PALAVRAS-CHAVE: suporte à decisão, conforto térmico, aves de postura.

\section{ESTIMATED THERMAL COMFORT CONDITION FOR LAYERS ACCORDING TO FUZZY THEORY}

\begin{abstract}
In this research a promising mathematical tool in system's analysis and/or process particularly in the area of Animal Production was used. Such tool was developed according to Fuzzy theory which in this specific case allows analyzing the composition of independent climatic variables, such as temperature and humidity that may influence the dependent variable named bird's thermal comfort. It was necessary to build up rules based on human intuition according to experts in this area, from which was possible to simulate distinct scenarios for supporting the decision of the construction of animal's housing. In this research thermal comfort for layer's housing in production were considered. The results were analyzed using the scientific computer environment of MATLAB 6.5, which could be done iteratively for each generated scenario. Based on the results it is possible to analyze thermal comfort conditions for several compositions of the input variables.
\end{abstract}

KEYWORDS: decision support, thermal comfort, layers.

\section{INTRODUÇÃO}

O sucesso na produção intensiva de animais está diretamente relacionado ao manejo eficiente do ambiente em que os mesmos estão inseridos. O controle do ambiente de alojamento é geralmente baseado em análises empíricas das medidas de temperatura e umidade relativa; entretanto, pesquisas apontam o potencial de usar análises teóricas dessas medidas e, assim, estabelecer critérios mais objetivos nas decisões dos produtores, como a baseada na teoria dos conjuntos Fuzzy (AMENDOLA et al., 2004), principalmente quando são incorporadas respostas fisiológicas dos animais a agentes estressores do ambiente, como, por exemplo, temperatura ambiente interferindo na resposta de temperatura corporal e produtividade (AERTS et al., 1996; GOEDSEELS et al., 1992; LACEY et al., 2000), o que, entretanto, insere maior complexidade nesse tipo de análise.

\footnotetext{
${ }^{1}$ Estudante de graduação, Faculdade de Engenharia Agrícola, UNICAMP, Campinas - SP. Bolsista do PIBIC/CNPq.

${ }^{2}$ Matemática, Professora Doutora, Faculdade de Engenharia Agrícola, UNICAMP, Campinas - SP.

${ }^{3}$ Eng ${ }^{a}$ Civil, Professor Titular Voluntário, Faculdade de Engenharia Agrícola, UNICAMP, Campinas - SP, irenilza@agr.unicamp.br Recebido pelo Conselho Editorial em: 17-2-2004

Aprovado pelo Conselho Editorial em: 7-6-2005
} 
A dificuldade de analisar o grande volume de informação referente a todas as variáveis envolvidas para o estabelecimento de condições adequadas na construção de galpão para animais vem sendo relatada na literatura afim, em geral, e em particular, nos trabalhos de pesquisa da área de Construções Rurais e Ambiência da FEAGRI/UNICAMP. QUEIRÓS et al. (2004) estimaram a tolerância térmica de suínos alojados em salas de creche, expostos a ambiente contendo amônia gasosa, enquanto MOURA et al. (2004) analisaram o conforto térmico em edificações para frangos de corte, variando o uso de ventilação forçada e sombreamento lateral.

Além disso, pesquisas com bovinos de leite, em áreas onde o conhecimento de especialistas fornece dados importantes para a consecução de tarefas, como, por exemplo, a inseminação artificial, relatam a melhoria de previsão de estro quando se utiliza da Teoria dos Conjuntos Fuzzy (FIRK et al., 2003). Ações, como pesagem de animais e dosagem de dietas utilizando a programação linear Fuzzy, associada a um processo de tomada de decisão, permitem a existência de mais combinações de soluções possíveis, diminuindo tanto o processo de leitura, como a redução do custo (CVETICANIN, 2003; CADENAS et al., 2003).

Um Conjunto Fuzzy A é caracterizado por uma função de pertinência, relacionando os elementos de um domínio, espaço, ou universo de discurso X, ao intervalo unitário [0,1] (ZADEH, 1965). Matematicamente, essa relação é descrita como: $\mu_{A}: X \rightarrow[0,1]$, em que o valor $\mu_{A}(x) \in[0,1]$ indica o grau com que o elemento $x$ de $X$ está no conjunto Fuzzy A, com $\mu_{A}(x)=0$ e $\mu_{A}(x)=1$ indicando, respectivamente, a não-pertinência e a pertinência completa de $x$ ao conjunto Fuzzy A.

A estrutura básica de um sistema baseado em regras Fuzzy inclui quatro componentes principais:

1) um fuzzificador, que traduz a informação de entrada em Conjuntos Fuzzy. A cada variável de entrada são atribuídos termos lingüísticos que são os estados da variável, e cada termo lingüístico é associado a um Conjunto Fuzzy traduzido por uma função de pertinência;

2) uma base de conhecimento, que contém um conjunto de regras Fuzzy (conhecido como base de regras) e um conjunto de funções de pertinência conhecido como base de dados;

3) um método de inferência, que aplica um raciocínio Fuzzy para obter uma saída Fuzzy, e

4) um defuzzificador, que traduz a saída por um valor numérico.

Com o objetivo de obter estimativas para as condições de conforto térmico no alojamento de galinhas poedeiras, este trabalho analisou como a composição das variáveis climáticas independentes, temperatura de bulbo seco e umidade relativa do ar, influencia na variável dependente aqui denominada de conforto térmico, em alojamentos de aves de postura comercial, a exemplo do que foi realizado para matrizes de frangos de corte, usando a Teoria dos Conjuntos Fuzzy.

\section{MATERIAL E MÉTODOS}

As variáveis independentes constituem intervalos de temperatura $\left({ }^{\circ} \mathrm{C}\right)$ e de umidade relativa do ar (\%) sobre os quais são definidas as variáveis lingüísticas. A variável dependente, denotada por conforto térmico, constitui intervalos, também classificados segundo o mesmo conceito.

A Tabela 1 foi organizada para os dados relativos ao alojamento de aves de postura. A variável dependente, denotada por conforto térmico, constitui intervalos, também classificados segundo o mesmo conceito. As classificações são formuladas segundo informações em LEESON \& SUMMERS (1997), ALBRIGHT (1990), WATHES (1994) e OLIVEIRA (1998). As informações disponíveis, que permitiram a construção da Tabela 1, não são excludentes e podem ser atualizadas constantemente, assim como os intervalos podem ser expandidos, desde que exista informação comprovada sobre os dados. 
TABELA 1. Classificação do estado de conforto térmico para aves de postura em fase de produção como função da temperatura de bulbo seco $\mathrm{T}\left({ }^{\circ} \mathrm{C}\right)$ e da umidade relativa do ar UR (\%).

\begin{tabular}{cccccc}
\hline U R $(\%)$ & \multicolumn{5}{c}{ Temperatura $\left({ }^{\circ} \mathrm{C}\right)$} \\
\hline$<70$ & $<12$ & $12-20$ & $20-25$ & $25-30$ & $>30$ \\
$70-75$ & ruim $(0,5)$ & médio $(0,6)$ & bom $(1,0)$ & bom $(0,6)$ & ruim $(0,7)$ \\
$75-80$ & ruim $(0,5)$ & bom $(0,9)$ & bom $(1,0)$ & bom $(0,5)$ & ruim $(0,7)$ \\
$80-85$ & ruim $(0,8)$ & bom $(0,9)$ & bom $(1,0)$ & médio $(0,5)$ & ruim $(0,8)$ \\
$>85$ & ruim $(0,8)$ & médio $(0,7)$ & bom $(0,9)$ & ruim $(0,6)$ & ruim $(1,0)$ \\
& ruim $(0,7)$ & médio $(0,7)$ & médio $(0,9)$ & ruim $(0,9)$ & ruim $(1,0)$ \\
\hline
\end{tabular}

A análise foi realizada segundo a teoria dos Conjuntos Fuzzy, no ambiente computacional MATLAB 6.5. O método de inferência utilizado foi o Método de Mamdani que combina os graus de pertinência referentes a cada um dos valores de entrada, por meio do operador mínimo, e agrega as regras por meio do operador máximo. A saída desse método é um conjunto Fuzzy. A defuzzificação foi feita usando o método do centro de gravidade, conforme sugerem AMENDOLA \& SOUZA (2004).

À medida que foram procedidas as análises para o cenário simulado, os valores da Tabela 1 foram alterados, em função das regras sugeridas por especialista.

\section{RESULTADOS E DISCUSSÃO}

Os termos lingüísticos foram atribuídos de acordo com as faixas mostradas na Tabela 1. Para a variável lingüística umidade relativa, foram considerados o domínio no intervalo $(65,90)$ e os termos: baixa, média baixa, média, média alta e alta (de acordo com os intervalos: <70; 70-75; 75-80; 80-85 e $>85$ ). As funções de pertinência selecionadas são trapezoidais, mas podem ser alteradas após consulta ao especialista. Na Figura 1, são mostradas as funções de pertinência estabelecidas para aves de postura.

Para a variável lingüística temperatura, foi considerado o domínio no intervalo $(7,35)$, representando as faixas da Tabela $1(<12 ; 12-20 ; 20-25 ; 25-30$ e $>30)$ pelos termos: muito baixa, baixa, média, alta e muito alta; também com funções de pertinência trapezoidais. Na Figura 2, verificam-se as funções de pertinência dos Conjuntos Fuzzy assumidas pela variável lingüística temperatura.

Para construir a base de regras, é necessário fuzzificar a variável de saída. Tomando-se por base as questões incertas, com relação aos limites do conforto ambiental para galinhas poedeiras em produção, que atualmente aparecem na literatura, foi criado um cenário para a variável de sensação de conforto térmico com os termos: ruim, médio e bom, num domínio $(0,1)$, de forma que o valor próximo do zero indica a pior sensação de conforto térmico, e perto do 1, a melhor. Nesse caso, resulta o que pode ser visto na Figura 3.

A Base de Regras composta por uma coleção de proposições Fuzzy, apresentadas na forma seentão, foi construída a partir da informação da Tabela 1 e com auxílio do especialista. Por exemplo, a primeira regra da Tabela 1 pode ser descrita como:

Se (UR é baixa) e (T é muito baixa) então (CT é ruim)

Os valores entre parênteses indicam o peso da informação, que pode estar em $(0,1)$, dependendo da indicação pelo especialista.

Na Figura 4, pode-se visualizar a variação não-linear da sensação de conforto térmico, como função da umidade relativa e temperatura de bulbo seco do ar e foi gerada a partir da Base de Regras. 


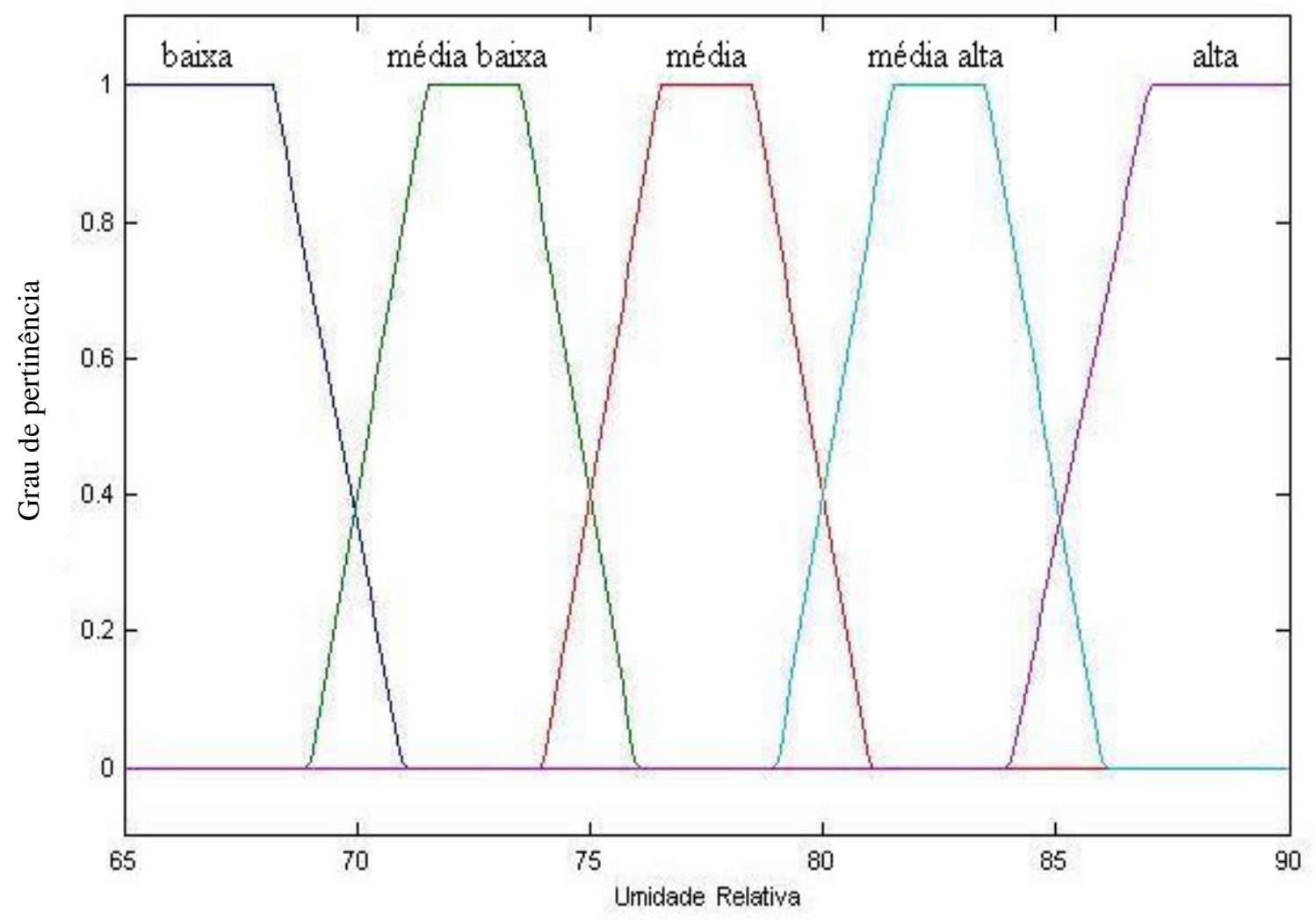

FIGURA 1. Funções de pertinência dos Conjuntos Fuzzy assumidos pela variável lingüística umidade relativa, para aves de postura.

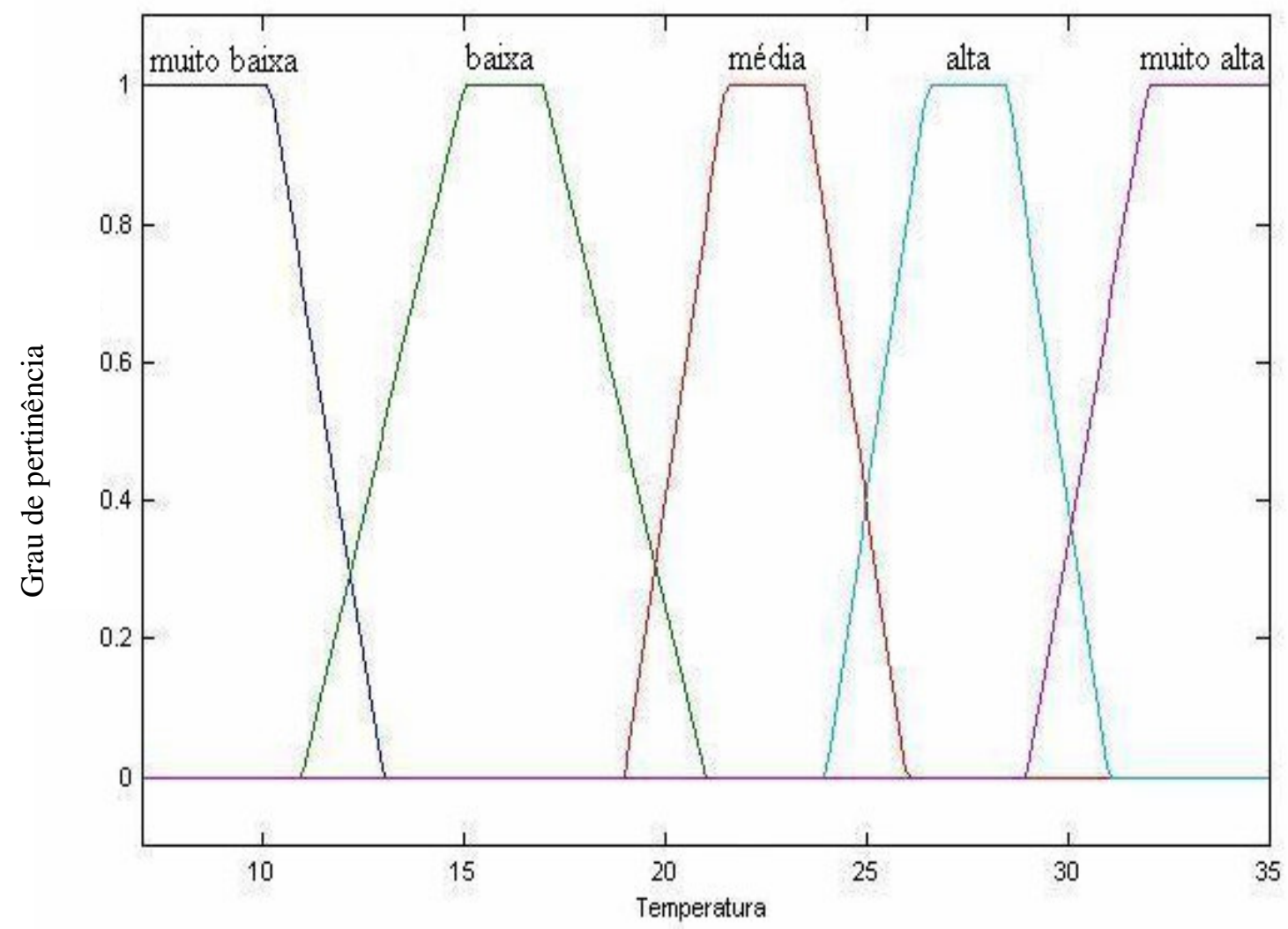

FIGURA 2. Funções de pertinência dos Conjuntos Fuzzy assumidos pela variável lingüística temperatura, para aves de postura. 


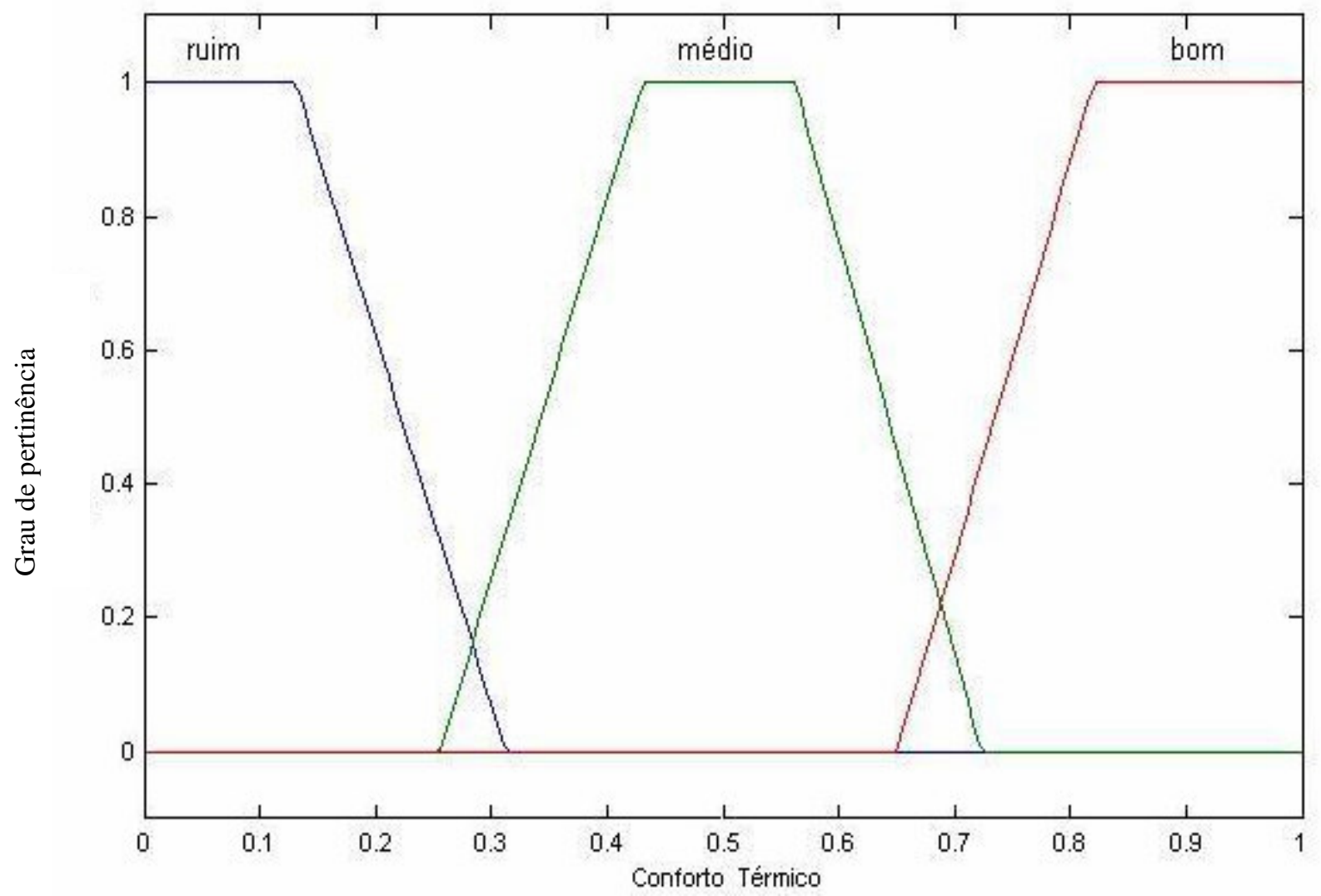

FIGURA 3. Funções de pertinência dos Conjuntos $F u z z y$ assumidos pela variável lingüística conforto térmico, para aves de postura.

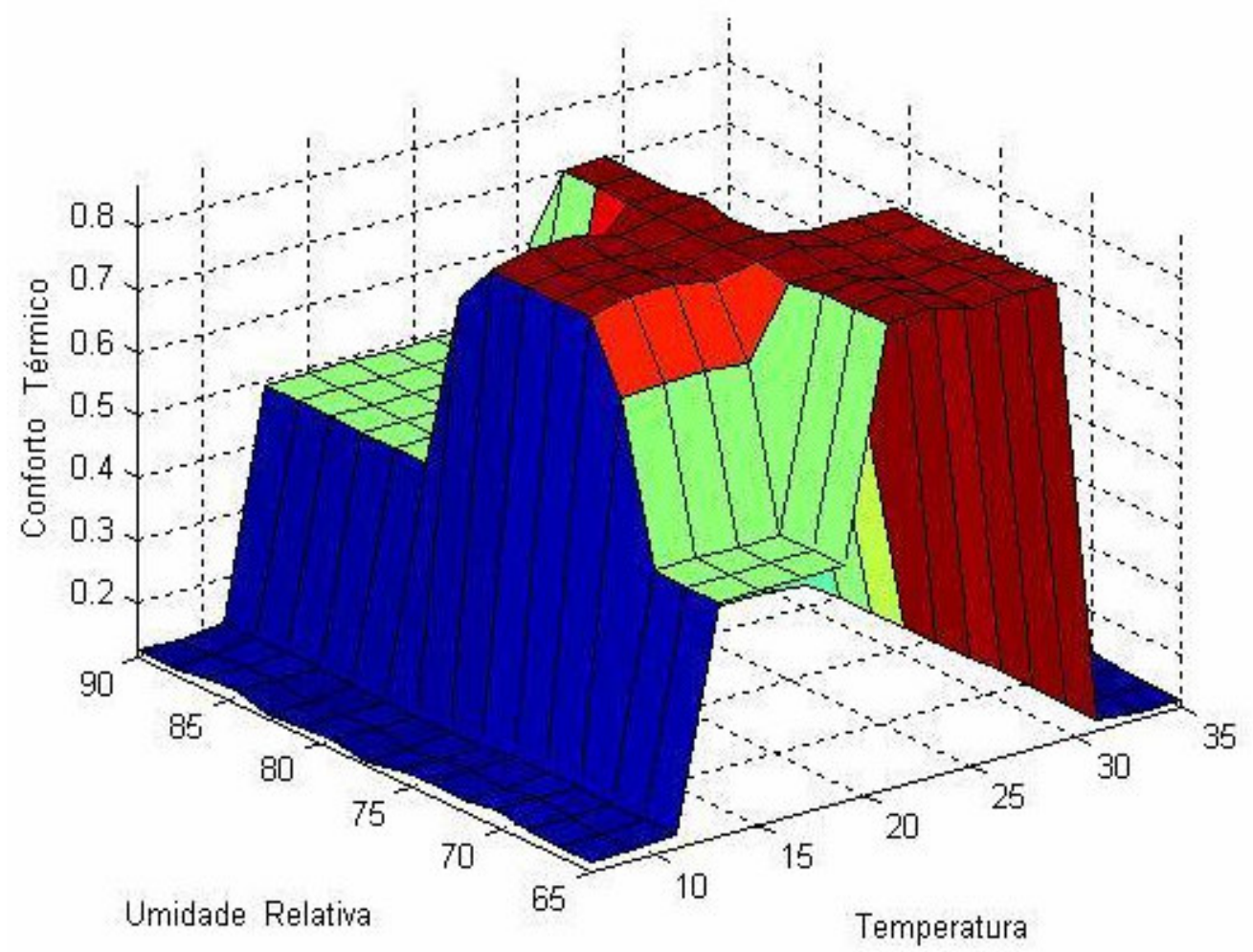

FIGURA 4. Estimativa da sensação de conforto térmico de aves de postura em produção, como função da temperatura e umidade relativa do ar. 
Dados os valores de temperatura de bulbo seco e umidade relativa do ar, tem-se como resultado a inferência de um valor de sensação de conforto térmico $(\alpha)$, no intervalo $(0,1)$ que representa a sensação de conforto térmico das galinhas poedeiras.

Na Figura 5, pode-se visualizar o cenário resultante da iteração da base de regras.

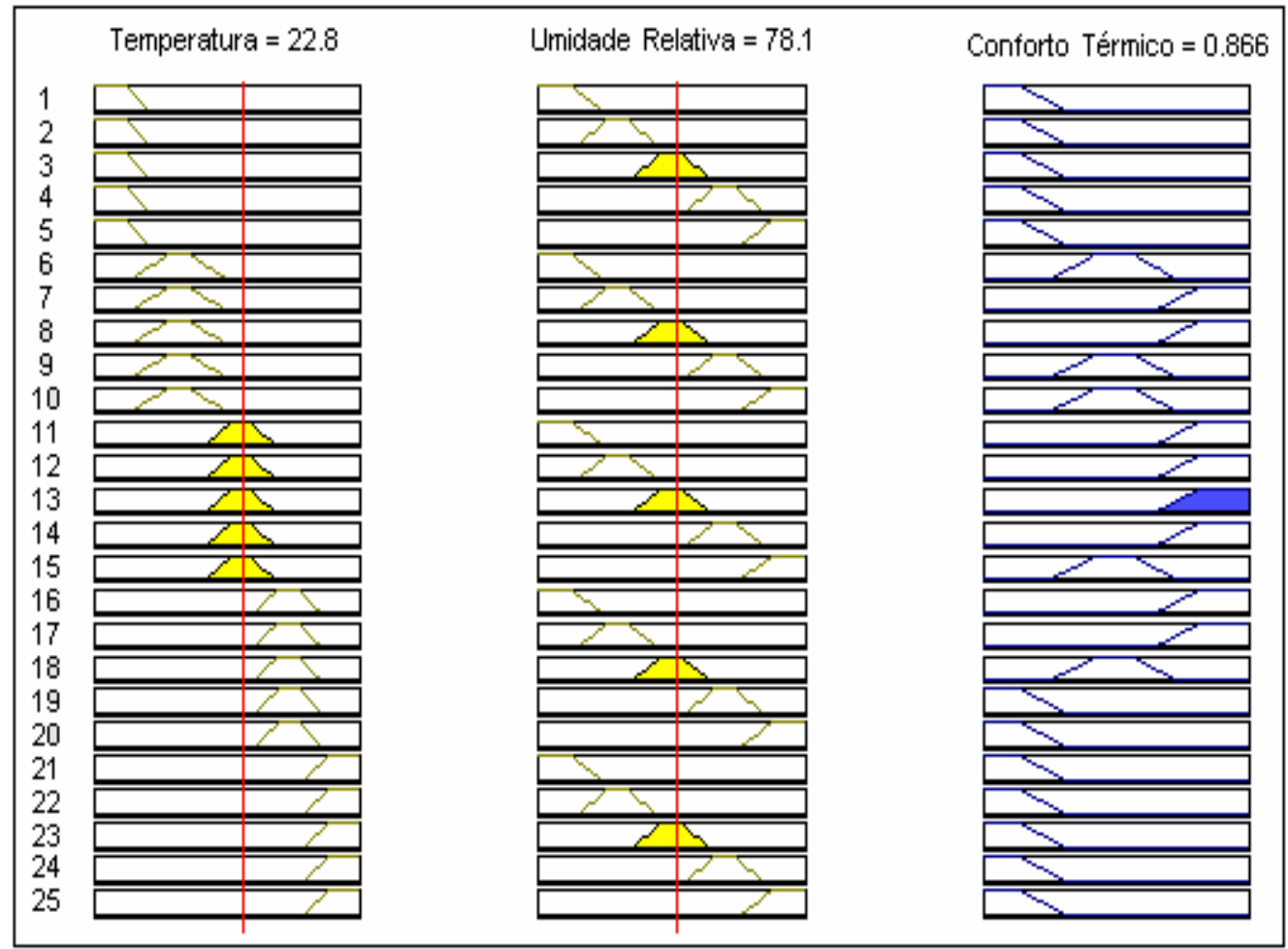

FIGURA 5. Base de Regras ativada para os valores de umidade relativa de $78,1 \%$, temperatura de bulbo seco igual a $22,8^{\circ} \mathrm{C}$ e resultado de conforto térmico para galinhas poedeiras em produção.

A partir do sistema de regras elaborado, foi gerada uma superfície que mostra a variação nãolinear da sensação de conforto térmico de frangos de corte, como função da temperatura de bulbo seco e da umidade relativa do ar do alojamento. Encontrou-se, para essas aves, a melhor situação de conforto térmico $\alpha \cong 0,866$, para a temperatura de bulbo seco de $22,8{ }^{\circ} \mathrm{C}$ e umidade relativa do ar de $78,1 \%$, que concorda com valores de termoneutralidade propostos por CURTIS (1983) e ALBRIGHT (1990).

Esses resultados indicam que é possível a estimativa de limites para a temperatura de bulbo seco e a umidade relativa do ar, em que a sensação térmica das aves alojadas seja máxima. A operacionalização desses resultados no suporte à decisão do controle da climatização do galpão, garantindo, assim, melhor produção, somente é possível por meio da manutenção da variável temperatura, uma vez que o controle da umidade relativa mostra-se operacionalmente complexo.

LACEY et al. (2000) também mostraram a viabilidade de predizer a temperatura corporal de aves correspondente a um certo acréscimo de temperatura ambiente, utilizando a Teoria de Conjuntos Fuzzy. Os autores comentam que esse procedimento, apesar de apresentar validação acurácia limitada, proporciona grande potencial de reduzir o impacto dos efeitos fisiológicos negativos, uma vez que permite antecipar a tomada de decisão dos sistemas de climatização.

Pesquisas futuras devem focar na incorporação de maior número de dados de ambiente e de produção, para compor um modelo mais robusto de predição. 
O uso dessa técnica pode contribuir com o avanço significativo da área de Construções Rurais e Ambiência, quando seus resultados forem associados aos dados de produção, o que já vem sendo estudado na Faculdade de Engenharia Agrícola da UNICAMP.

\section{CONCLUSÕES}

Utilizando a Teoria dos Conjuntos Fuzzy, no ambiente de computação científica MATLAB 6.5, foi possível estimar a sensação de conforto térmico de galinhas poedeiras em produção, com relação à temperatura de bulbo seco e à umidade relativa do ar.

\section{REFERÊNCIAS}

AERTS, J.M.; BERCKMANS, D.; SCHUMMANS, B. On-line measurement of bioresponses for climate control in animal production units. In: INTERNATIONAL CONFERENCE ON COMPUTERS IN AGRICULTURE, 6., 1996, Michigan. Proceedings...St Joseph: American Society of Agricultural and Biological Engineering, 1996. 147-53p.

ALBRIGHT, L.D. Environmental control for animals and plants. St. Joseph: ASAE, 1990.276 p.

AMENDOLA, M.; CASTANHO, M.J.; NÄÄS, I.A.; SOUZA, A.L. Análise matemática de condições de conforto térmico para avicultura usando a teoria dos conjuntos Fuzzy. Biomatemática Brasil, Campinas, v.14, p.87-92, 2004.

AMENDOLA, M.; SOUZA, A. Manual do uso da teoria dos conjuntos Fuzzy no MATLAB 6.5. Campinas: CPG/FEAGRI/UNICAMP, 2004. 30p.

CADENAS, J.M.; PELTA, D.A.; PELTA, H.R.; VERDEGAY, J.L. Application of Fuzzy optimization to diet problems in Argentinean farms. Disponível em: <http://www.sciencedirect.com>. Acesso em: 5 jul de 2003.

CURTIS, S.E. Environmental management in animal agriculture. Ames: The Iowa State University Press, 1983. 409 p.

CVETICANIN, D. New approach to the dynamic weighing of livestock. Biosystems Engineering, United Kingdon, v.86, n.3, p.247-52, 2003.

FIRK, R.; STAMER, E.; JUNGE, W.; KRIETER, J. Improving oestrus detection by combination of activities measurements with information about previous oestrus cases. Livestock Production Sciences, Clare, v.82, n.1, p.97-103, 2003.

GOEDSEELS, V.; GEERS, R.; TRYEN, B.; WOUTERS, P.; GOOSSENS, K.; VILLE, H.; JANSSENS, S. A data acquisition system for electronic identification, monitoring, and control of group-housed pigs. Journal of Agricultural Engineering, Silsoe, v.52, May-August, p.25-33, 1992.

LACEY, B.; HAMRITA, T.K.; MCCLENDON, R. Feasibility of using neural networks for a real-time prediction of poultry deep body temperature responses to stressful changes in ambient temperature. Applied Engineering in Agriculture, St Joseph, v.16, n.3, p.303-8, 2000.

LEESON, S.; SUMMERS, J.D. Commercial poultry nutrition. Guelph: University Books, 1997. 303 p.

MATLAB® 6.5. The Mathworks Inc. 2004. 03 Apple Hill Drive. Natick, MA 01760-2098. Disponível em: http://www.mathworks.com/. Acesso em: 17 jan. 2004

MOURA, D.J.; NÄ̈̈S, I.A.; QUEIROZ, M.P.G. Estimating thermal comfort and solar orientation in broiler housing using Fuzzy Logic. In: CONGRESO LATINOAMERICANO Y DEL CARIBE DE INGENIERIA AGRÍCOLA, 6., 2004, San José, Costa Rica. Memória... San José: Asociación Costaricense de Ingenieros Agrícolas, 2004. 1 CD-ROM. 
SILVA, I.J.O. Desenvolvimento de modelos matemáticos para análise da influência das condições ambientais na produção industrial de ovos. 1998. 103 f.Tese (Doutorado em Engenharia Agrícola) Faculdade de Engenharia Agrícola, Universidade Estadual de Campinas, Campinas, 1998.

WATHES, C.M. Air and surface hygiene. In: WATHES C. M.; CHARLES D. R. Livestock housing. Wallingford: CAB International, 1994. p.123-48.

ZADEH, L. A. Fuzzy Sets. Information and Control, United Kingdom, v.8, p.338-53, 1965. 